\title{
A Cancellation Method for Background Noise in Sound and Electromagnetic Environments by Using a Digital Filter for Power State Variables
}

\author{
Akira Ikuta ${ }^{\dagger}$ \\ Faculty of Human Life and Environmental Science, Hiroshima Prefectural Women's University, 1-1-71 Ujina-Higashi, \\ Minamiku, Hiroshima, 734-8558 Japan
}

\author{
Mitsuo Ohta ${ }^{\dagger}$ \\ Hiroshima University, Emeritus, 1105, 1-7-10 Matoba, Hiroshima, 732-0824 Japan
}

(Received 15 October 2002; accepted 14 March 2003)

\begin{abstract}
The observed phenomena in real wave motion type environments (sound, vibration, and electromagnetic) are inevitably contaminated by the background noise of arbitrary distribution and type. Therefore, in order to evaluate a wave motion type environment, it is necessary to establish some estimation methods to remove the undesirable effects of the background noise. In this paper, we propose a digital filter for estimating the power state variable of a specific signal with the existence of background noise of non-Gaussian distribution form. By applying the well-known least-mean-square method for the moment statistics with several orders, a practical method for estimating the specific signal is derived. The effectiveness of the proposed theoretical method is experimentally confirmed by applying it to estimation problems in real sound and magnetic field environments.
\end{abstract}

${ }^{\dagger}$ Member of the International Institute of Acoustics and Vibration (IIAV)

\section{INTRODUCTION}

The specific signal in real wave motion type environments, such as those created by sound, vibration, and electromagnetic waves, frequently exhibits some very complex fluctuation forms of a non-Gaussian type, owing to natural, social and human factors. ${ }^{1,2}$ Furthermore, the observed data are inevitably contaminated by the background noise of arbitrary distribution and type., ${ }^{3,4}$ In these situations, it is often desirable to estimate several evaluation quantities, such as $L_{e q}$ (averaged energy on a decibel scale $), L_{x}((100-x)$ percentile level, $x=5,10,50,90,95)$, the peak value, the amplitude probability distribution, the average crossing rate, the pulse spacing and duration distributions, etc. of the specific signal. Without losing their mutual relationships, it is indispensable to estimate the original wave fluctuation form itself of the specific signal based on the observed noise data.

Hitherto, many methodological studies have been reported on the state estimation for stochastic systems. ${ }^{\mathbf{5 , 6}}$ However, many standard estimation methods proposed previously in studies of stochastic systems are restricted only to Gaussian distributions (in most cases, zero mean) ${ }^{7,8}$ Real wave motion type environments often show an intricate fluctuation pattern rather than the standard Gaussian distribution. For example, since the specific signal in sound environment systems is usually measured with a sound level meter, the sound environment very often may be considered on an energy scale as a system with a signal of non-zero mean. So, it becomes essentially a big problem to apply the conventional state estimation methods to the present situation without any improvement to them.

In our previous studies, ${ }^{\mathbf{9}, 10}$ several state estimation methods for a stochastic environment system with non-Gaussian fluctuations have been proposed based on expansion expressions for the probability distribution. Since our previously reported estimation algorithms were based on the whole of the probability distribution, their derivation processes became rather complicated.

In this study, a dynamic method for estimating a specific signal with the existence of background noise with a nonGaussian distribution form is proposed in a recursive form suitable for use with a digital computer. More specifically, by paying attention to the power state variable for a specific signal in a wave motion type environment, which exhibits complex probability distribution forms, we propose a new type of digital filter for estimating a specific signal on a linear power scale. In the case of considering the power state variable, a physical mechanism of contamination by background noise can be reflected in the state estimation algorithm by using the additive property between the specific signal and the background noise. A Laguerre polynomial is suitable to represent the power state variable, which fluctuates only within the positive region. The proposed digital filter positively utilises the additive property of power state variables in the estimation algorithm. Instead of focusing on the whole of the probability distribution in our previous studies, ${ }^{9,10}$ by applying the well-known least-mean-square method for the moment statistics with several orders, a simplified estimation algorithm is derived from a practical viewpoint.

The proposed method can be applied to the assessment and regulation of environmental noise such as road traffic and factory noise. Because background noise always exists in the measurement of environmental noise, the effects of the background noise must be removed from the measured data in order to evaluate the environmental noise. Furthermore, in the actual living and/or working environment, various electronic equipment exists, which generates electromagnetic 